\title{
SUPUESTOS, LÍMITES Y ALCANCES DE LA FILOSOFÍA INTERCULTURAL
}

Raúl Fornet-Betancourt

Missionwissenschaflichen Institut. Aachen. Alemania

\section{Algunas aclaraciones mínimas}

La historia de la filosofía -sobre todo la que se nos trasmite por la historiografía académica- no da, seguramente, la medida integral de la filosofía; sin embargo, creo que quien consulte dicha historia de la filosofía, aunque sea sólo de forma somera, puede extraer de ella el convencimiento fundado de que la filosofía hace buena parte de su historia en base a procesos de transformación por los que se reubica tanto teórica como contextualmente. De modo que se puede decir que la historia de la filosofía es (también) la historia de sus transformaciones.

He querido comenzar con esta indicación para situar la filosofía intercultural en esa historia de las transformaciones de la filosofía. Es decir que lo primero que deseo resaltar, al hablar de este proyecto que se conoce o resume hoy con el nombre de «filosofía intercultural», es que se trata, fundamentalmente, de una propuesta programática para una nueva transformación de la filosofía. Se busca una nueva configuración de la filosofía o, mejor dicho, del filosofar; ya que no se pretende simplemente una reconfiguración de la filosofía como disciplina académica sino también una renovación de la actividad filosófica, del quehacer filosófico en general, teniendo en cuenta precisamente las distintas prácticas del filosofar con que nos confrontan las culturas de la humanidad. Pero, como sobre este punto volveré luego al hablar de los supuestos de la filosofía intercultural, paso al segundo aspecto que me parece necesario destacar de entrada. 
Lo segundo que debe quedar claro cuando hablamos de filosofía intercultural, es que ésta, como se desprende del primer aspecto señalado, no representa una nueva área temática que vendría a complementar las disciplinas específicas de la filosofía tradicional, como lo hizo en su día, por ejemplo, la filosofía de la técnica; y por eso tampoco se la debe confundir con una expresión filosófica que hoy vuelve a tomar auge y que parece coincidir con ella, a saber, la filosofía de la cultura. Pues la filosofía intercultural, aunque también lo hace, no tiene su eje de desarrollo ni su preocupación central en el análisis de las culturas o en el intento de facilitar una compresión filosófica de las culturas. Su atención se centra más bien en la búsqueda de pistas culturales que permitan la manifestación polifónica de lo que llamamos filosofía desde el multiverso de las culturas.

Y lo tercero que quiero aclarar de entrada es que la filosofía intercultural no es un fenómeno aislado, producto de algunos filósofos que quieren lanzar una nueva moda, sino que hay que comprenderla como parte articulada en un movimiento multidisciplinar e internacional de pensadores e investigadores que tratan de afrontar uno de los mayores desafíos que nos plantea nuestro mundo histórico actual: el desafío de la convivencia solidaria -iy no sólo pacífica!- entre seres humanos de la más distinta procedencia cultural, y vinculados, en gran parte, a tradiciones e identidades religiosas fuertes, en un mundo marcado por estrategias globalizadoras que los "acerca» (sobre todo virtualmente) y que los «engloba» como «destinatarios» de todo tipo de «mensajes» en la «red», pero que no necesariamente los comunica en tanto que sujetos que se reconocen y que se hacen prójimo'.

De suerte que la filosofía intercultural se enmarca en un movimiento alternativo de gran alcance que persigue, en suma, un objetivo doble; pues, por un lado, se trabaja en la cristalización de un cambio de paradigma a nivel "teórico» o "científico» que permita no sólo una nueva constelación de los saberes de la humanidad sino también un diálogo abierto a escala mundial sobre los ideales (¿valores?) que deben guiar nuestra investigación científica o, dicho más sencillamente, sobre lo que queremos y/o debemos saber realmente; y, por otro lado, se trata de complementar ese giro paradigmático a nivel «teórico» con la propuesta práctica de reorganizar el mundo globalizado haciendo valer, contra las fuerzas uniformizantes y niveladoras de la globalización actual dominante, que en el mundo hay pueblos que hacen mundo en plural y que el futuro de la humanidad, por tanto, también puede ir por el rumbo de la solidaridad entre mundos reales que se respetan, esto es, de una humanidad solidaria conviviente en muchos mundos.

Por eso este movimiento se expresa hoy, con igual fuerza, tanto a nivel de lo que podríamos llamar la producción científica, como a nivel práctico-político. Es evidente que en el marco de este breve estudio no me puedo demorar en la presentación detallada de las múltiples formas de expresión de este movi-

\footnotetext{
${ }^{1}$ Cf. RICOEUR P., Histoire et vérité, Paris 1955, especialmente pp. 99 y sgs.
} 
miento. Pero, para ilustrar al menos en forma ejemplar su dimensión multidisciplinar, me permito anotar algunas de las áreas donde el movimiento intercultural alcanza hoy expresiones muy representativas: Antropología ${ }^{2}$, Comunicación ${ }^{3}$, Derecho ${ }^{4}$, Educación $^{5}$, Filología ${ }^{6}$, Pedagogía7, Psicología ${ }^{8}$ o Teología 9 . Y por lo que hace a sus expresiones en el nivel práctico-político baste ahora con remitir a su presencia e incidencia en foros internacionales de la UNESCO ${ }^{10}$ o del Foro Internacional de las Alternativas ${ }^{11}$.

${ }^{2}$ Cf. BONFIL BATALLA Guillermo (Comp.), Hacia nuevos modelos de relaciones interculturales, México 1991; DIETZ Gunther, El desafío de la interculturalidad, Granada 2000; HÖRNING Karl H/ WINTER Rainer (Ed.), Widerspenstige Kulturen, Frankfurt/M. 1999; y ROSALES AYALA Hector (Coord.), Cultura, sociedad civil y proyectos culturales en México, México 1994.

${ }^{3}$ Cf. GARCEA E.A., La comunicazione interculturale, Roma 1996; APFELBAUM Birgit/MÜLLER Hermann (Ed.), Fremde im Gespräch, Frankfurt/M. 2000; AITHAL V. /SCHIRILLA N. /SCHÜRINGS H.MEBWE S. (Ed.), Wissen - Macht - Transformacion, Frankfurt/M. 1999; y WISEMAN R.L., Intercultural Comunication Theory, Los Angeles 1995.

${ }^{4}$ Cf. ALMEIDA Ileana/ARROBO Nidia (Ed.), En defensa del pluralismo y la igualdad, Quito 1998; DE LUCAS J., «Derechos humanos-Legislación-Interculturalidad», en Revista de Documentación Social 25 (1994) 73-90; ORSI G. /SEELMANN H. /SMID S. /STEINVORTH U. (Ed.), Recht und Kulturen, Frankfurt/M. 2000; PÉREZ TAPIA José A., «¿ldentidades sin frontera? Identidades particulares y derechos humanos universales», en GÓMEZ GARCÍA P. (Coord.), Las ilusiones de la identidad, Madrid 2000, pp. 55-98; y STAVENHAGEN Rodolfo, «Derechos indígenas y derechos culturales de los pueblos indígenas», en KLESING-REMPEL Ursula (Comp.), Lo propio y lo ajeno, México 1996, pp. 71-96; sin olvidar los trabajos de J. Habermas y O. Höffe.

${ }^{5}$ Cf. ALBÓ Xavier, Iguales aunque diferentes, La Paz 1999; Centro Boliviano de Investigación y Acción Educativos (Ed.), Diversidad Cultural y procesos educativos, La Paz 1998; KLESING REMPEL Ursula (Comp.), Lo propio y lo ajeno. Interculturalidad y sociedad multicultural, México 1996; y ZÚÑIGA M./ ANSIÓN J., Interculturalidad y educación en el Perú, Lima 1997.

${ }^{6}$ Cf. HAMMERSCHMIDT Annette C., Fremdverstehen, München 1997; LENKERSDORF Carlos, Los hombres verdaderos. Voces y testimonios tojolabales, México 1996; y WIERLACHER Alois (Ed.), Das Fremde und das Eigene, München 1985.

${ }^{7}$ Cf. HEISE Maria (Ed.), Interculturalidad. Creación de un concepto y desarrollo de una actitud, Lima 2001; NIEKRAWITZ Clemens, Interkulturelle Pädagogik im Überblick, Frankfurt/M. 1990; asî como el anuario «Pädagogik: Dritte Welt», Frankfurt/M., editado por Patrick V. DíAS, uno de los pioneros de la pedagogía intercultural.

${ }^{8}$ Cf. PELTZER Karl, Psychology and Health in African Cultures, Frankfurt/M. 1995; THOMAS Alexander, Kulturvergleichende Psychologie, Göttingen 1994; y THOMAS A. (Ed.), Psychologie interkulturellen Handelns, Göttingen 1996.

${ }^{9}$ Cf. ELIZONDO Virgilio, "Condiciones y criterios para un diálogo teológico intercultural», en Concilium 191 (1984) 41-51; KESSLER Hans (Ed.), Ökologisches Weltethos im Dialog der Kulturen und Religionen, Darmstadt 1996; y SCHREIJÄCK Thomas (Ed.), Menschwerden im Kulturwandel, Luzern 1999. Cabe destacar que la Facultad de Teología Católica de la Universidad de Francfort ofrece una especialidad en "Theologie interkulturell» y que edita además una serie de monografías con ese título.

${ }^{10} \mathrm{Cf}$. UNESCO (Ed.), Introduction aux études interculturelles, Paris 1980.

"Cf. «Manifiesto del Foro Internacional de las Alternativas», en Pasos 76 (1998) 33-35. Ver también FORNET-BETANCOURT Raúl, «Interkulturelle Beziehungen als Gegenmodell zur Globalisierung», en OEKOS (Ed.), Globalisierter Markt-Ausgeschlossene Menschen, Sankt Gallen 1997, pp. 15-17; y SIDEKUM Antonio (Ed.), Corredor de Idéias. Integração e globalização, São Leopoldo 2000. 
De este movimiento nos interesa ahora, como es lógico, sólo el área de filosofía que no se ha nombrado antes precisamente porque es objeto de este estudio. Paso, por tanto, a presentar algunos momentos centrales de la filosofía intercultural como perspectiva de trabajo para reorientar el filosofar ${ }^{12}$.

\section{Algunos supuestos, o esbozando el punto de partida de la filosofía inter- cultural}

En el punto anterior mencioné de pasada «las distintas prácticas del filosofar con que nos confrontan las culturas de la humanidad», señalando que volvería sobre esta afirmación en este segundo apartado. Pues bien, quiero retomarla ahora para explicitar lo que considero el primer supuesto de la filosofía intercultural: la experiencia de las culturas de la humanidad como lugares donde se practica la filosofía. Hay, pues, «filosofía» no porque haya un modelo paradigmático que se expande y globaliza (me refiero al «mito» de Grecia como único lugar de nacimiento de la filosofía), sino porque hay "prácticas culturales» de filosofía como ejercicio concreto de pensar que se hace cargo de su contextualidad e historicidad.

El supuesto de que las culturas son lugares filosóficos, lugares que posibilitan prácticas específicas de eso que se llama filosofía, implica que se parte de la necesidad de desoccidentalizar la filosofía desde su origen, es decir, "desde la cuna»; pero no por un afecto antioccidental ni para regatearle méritos a Occidente, ya que esa desoccidentalización significa simplemente un poner a Occidente en su lugar; es decir, verlo como un lugar de tradiciones complejas que, desde siempre, ha estado en relación con otros mundos y que, en cuanto tal, no es el lugar de toda la filosofía posible sino el lugar de ciertas posibilidades de filosofía.

Esta relativización de la localización del origen occidental de la filosofía implica a su vez un segundo supuesto. Me refiero, de hecho, a algo que se puede considerar como una consecuencia directa del primer supuesto; a saber, que la filosofía es también plural en sus formas de articularse, de organizarse o de institucionalizarse. Se supone, en efecto, que la pluralidad de prácticas culturales de la filosofía, además de remitir a una multiplicidad de lugares de nacimiento, es fuente de diferencias en las formas concretas por las que se va desarrollando el quehacer filosófico y, con ello, también en el nivel de constitución de las referencias tradicionales para discernir qué es lo que vale o se reconoce como «filosofía» en un determinado universo cultural.

De aquí, pues, que la filosofía intercultural crea necesario regionalizar la validez de las formas de expresión filosófica sancionadas por la tradición occi-

\footnotetext{
${ }^{12}$ Como aquí nos concentramos sobre todo en la explicitación de las bases filosóficas comunes no entraremos en el análisis de las distintas posiciones que ya hoy se pueden distinguir en la «filosofía intercultural». Para ello ver el excelente trabajo de DE VALLESCAR Diana, Cultura, Multiculturalidad e Interculturalidad. Hacia una racionalidad intercultural, Madrid 2000.
} 
dental dominante, tales como, por ejemplo, el curso académico, la publicación del tratado o libro especializado, la conferencia en los gremios filosóficos profesionales o la agrupación en sociedades de especialistas; para abrir el horizonte del quehacer filosófico a otras formas de ejercicio y de reconocimiento, como podrían ser, por ejemplo, el discurso oral o la articulación contextual en la vida cotidiana.

En conexión con esto está el tercer supuesto que puede resumirse diciendo que la filosofía intercultural, en parte como reacción a la reductora comprensión de la filosofía como "ciencia» en el sentido (monocultural) occidental, parte de la necesidad de «mundanizar» la filosofía. Y debo aclarar, para evitar malentendidos o comparaciones con propuestas que pueden parecer similares -pienso concretamente en la filosofía popular de la ilustración o en el programa del joven Marx- que no se trata de encarnar ni de popularizar la filosofía. «Mundanizar» quiere decir aquí más bien contextualizar, pero precisamente no como una propiedad que el quehacer filosófico adquiere a posteriori sino como el humus desde el que crece. Se trata, dicho con otras palabras, de un supuesto que postula la dignificación de los contextos culturales al considerarlos universos específicos, y no fragmentos; mundos que, por dar en qué y cómo pensar, producen filosofías de y para mundos contextuales. De aquí que, a otro nivel, el supuesto de «mundanizar» la filosofía conlleve a preferir la práctica de la filosofía en tanto que saber que sabe hacer realidades; y que no se queda, por consiguiente, en un mero saber de realidades.

El cuarto supuesto, que está relacionado con el anterior, tiene que ver con la comprensión de la universalidad en filosofía. Pues la filosofía intercultural no es postmoderna, ni tampoco «contextualista» en el sentido de Rorty. Es decir que sigue suponiendo la necesidad de la universalidad. Su apuesta por las filosofías contextuales no es, en modo alguno, renuncia a la universalidad y a la comunicación; pero sí rechazo de una universalidad constituida sobre la base de la diferencia entre lo universal y lo particular y frente a la cual lo particular siempre necesita justificarse porque lo universal se presenta como el orden regulador de la convivencia o expresión de la medida de lo humano. Para la filosofía intercultural esta universalidad constituida tiene que ser criticada. Mas su crítica no cuestiona lo universal o la pretensión de universalidad que hay en ella. Lo que realmente se critica es su constitución en un orden con límites definidos y, por tanto, excluyentes; su proceso de cristalización en las fronteras de un universo cultural determinado, el occidental; y como resultado de ordenamientos estabilizadores y disciplinadores de alternativas propias, como pueden ser la dominante patriarcal o la configuración «burguesa» de la cultura. Se critica, en suma, no lo universal sino las deficiencias en universalidad que conlleva este modelo.

Por otra parte, la filosofía intercultural prefiere replantear la cuestión de la universalidad sustituyendo la dialéctica de la tensión entre lo universal y lo particular por el culto (cultivo) del diálogo entre universos contextuales que testimonian su voluntad de universalidad con la práctica de la comunicación. Y hay que añadir que esta práctica de la comunicación, por ser justo ejercicio con- 
textual que busca trasmitir las experiencias y referencias fundantes de sus universos respectivos, es ante todo un esfuerzo de traducción. Los universos culturales se traducen, y traduciéndose unos a otros van generando universalidad. Se apuesta, en una palabra, por un nuevo tipo de universalidad cuya imagen más adecuada sería acaso la de una «escuela de traducción» sin numerus clausus y en permanente actividad.

Por último quisiera nombrar el supuesto que concierne explícitamente a la concepción de la razón con la que trabaja la filosofía intercultural. Pues, como se deduce de lo que se ha dicho hasta ahora, es evidente que la filosofía intercultural tiene fuertes sospechas frente al modelo de razón que normalmente se suele presentar como paradigma de toda reflexión filosófica que quiera merecer este nombre. En analogía con la crítica de la universalidad constituida hay que observar, sin embargo, que la filosofía intercultural no sospecha de la razón filosófica usual ni por «racional» ni por «filosófica» sino por la monoculturalidad occidental («occidental» se refiere aquí a la tradición cultural dominante que se impone en los procesos de institucionalización y que resulta opresora incluso para otras tradiciones también occidentales) que reflejan sus definiciones de ambos términos. Es la hipoteca de ser resultado de un proceso de constitución regulado en gran parte por el monólogo del Occidente (reducido) consigo mismo, lo que hace sospechosa la configuración de la razón filosófica que muchos defienden todavía como encarnación de universalidad por excelencia. Frente a este «uso» de la razón filosófica la filosofía intercultural propone historificar el proceso de constitución de las formas de racionalidad vigentes; revisarlas desde un diálogo intercultural, abierto y sin prejuicios, que permita hacer manifiesto el tejido monocultural de dicho proceso y corregirlo con un plan de reconstrucción de la razón filosófica desde y con la participación traductora de las prácticas del quehacer filosófico en las distintas culturas de nuestro multiverso.

En un plano más concreto, y de cara al trabajo inmediato, este supuesto implica para la filosofía intercultural superar el marco referencial de la razón sistemática y sistematizada o, más exactamente, de la razón arropada con sistemas que, por muy elaborados que sean, no pueden, debido justo a la contextualidad e historicidad de los modos de saber y de hacer, dar la medida acabada de la razón como comunión de prácticas de razón. Todo intento dentro de ese marco referencial parece llevar a formalismos sacrificadores de la materialidad de la vida y de las culturas; y con ello, curiosamente, a la pérdida de la capacidad crítica en el quehacer filosófico ${ }^{13}$. Para superar este horizonte de la razón (¡sin negarlo!), la filosofía intercultural propone un programa de reubicación de la razón desde la consulta abierta de sus usos plurales en las prácticas culturales de la humanidad, tanto pasadas como presentes. Todas las culturas conocen procesos de discernimiento y prácticas de diferenciación inter-

${ }^{13}$ Cf. DUSSEL Enrique, Etica de la liberación en la edad de la globalización y de la exclusión, Madrid, Trotta, 1998. 
na; procesos y prácticas que son a su vez reflejo de conflictos internos; es decir, expresiones del «litigio» intracultural entre fuerzas sociales con diferentes intereses de poder, políticos, económicos, genéricos, religiosos, etc., que luchan precisamente por fundar tradición en su universo cultural y crear así referencias vinculantes para el ordenamiento del mismo ${ }^{14}$. Suponiendo, pues, que procesos semejantes se dan en todas las culturas y que son ellos los que explican la formación histórica de lo que llamamos tradiciones culturales, pero también el trato con las tradiciones, la filosofía intercultural propone rehacer la historia de la razón (filosófica) desde la relectura de esos procesos y de esas prácticas contextuales porque entiende que son el lugar donde se van cristalizando los modos en que el género humano aprende a dar razón de su situación en un universo concreto (dimensión contextual) y a razonar, con las razones de los otros, sobre lo que mejor conviene a todos para realizar en todos los contextos la humanidad de todos (dimensión universalizante).

La propuesta de la reubicación de la razón (filosófica) es, por tanto, una propuesta de ensanchar las fuentes de razonamiento, así como sus expresiones; y abriga, por consiguiente, la esperanza de una transformación radical de la razón (filosófica). Sería, dicho en una frase, búsqueda programática de una configuración de la razón como tejido de modelos de razonamientos mediante un proceso de aprendizaje intercultural que amplia los criterios de discernimiento y compensa las unilateralidades de la razón en su figura histórica de razón sometida a la dinámica del desarrollo cultural-civilizatorio de la modernidad occidental-capitalista ${ }^{15}$.

\section{Límites y alcances de la filosofía intercultural}

Mientras que en varios de los campos señalados más arriba, como la pedagogía o la antropología, el movimiento alternativo de la interculturalidad cuenta ya con resultados avalados por una práctica de años (y articulados además en muchos casos como ejes centrales en políticas culturales) que permiten evaluar con cierto fundamento los límites y alcances reales del planteamiento intercultural, en filosofía nos encontramos con una situación un tanto diferente por cuanto que el desarrollo explícito en esta área es mucho más reciente y las perspectivas de trabajo propuestas están todavía en plena evolución. De modo que parece todavía un poco prematuro y arriesgado hablar de «límites y alcances de la filosofía intercultural». Con todo, y por respetar el título con el que se me pidió este artículo, intentaré delinear algunos momentos del desarrollo de la filosofía intercultural en base a los cuales se pueden inducir sus

\footnotetext{
${ }^{14}$ Dejo anotado que éste sería el lugar para analizar la diferencia entre «cultura» y «sociedad», ver las formas de interacción entre ambos niveles y plantear la cuestión de si las culturas, por la tendencia integrativa que les es constitutiva, no son el foco que mantiene viva la posibilidad de la «relación comunitaria», de la comunidad, en las sociedades complejas de hoy.

${ }^{15}$ Una presentación más detallada de los supuestos mencionados aquí puede verse en FORNETBETANCOURT Raúl, Transformación intercultural de la filosofía, Bilbao, 2001.
} 
alcances y límites, pero subrayando que no se trata de un balance sino de una aproximación provisional.

Haciendo ahora abstracción de la importancia, sin duda, decisiva de muchos trabajos pioneros de distintos autores ${ }^{16}$, se puede decir que el desarroIlo de la filosofía intercultural toma forma orgánica y proyección internacional entre 1991 y 1995, con la fundación de la «Gesellschaft für Interkulturelle Philosophie» (1991), con sede en Colonia; con la aprobación de la «Wiener Gesellschaft für Interkulturelle Philosophie» (1994), que tiene su sede en Viena; y con el comienzo de la iniciativa, coordinada por el Departamento de América Latina del Missionswissenschaftliches Institut en Aachen, de los «Congresos Internacionales de Filosofía Intercultural», en 1995, en México. Pues con la creación de estos tres focos el desarrollo de la filosofía intercultural supera el marco del empeño personal de pensadores aislados para convertirse en el objeto de una actividad coordinadora internacionalmente, y que busca, además, asegurar su continuidad estableciendo los canales institucionales adecuados. Pero lo decisivo es que estos tres focos representan en cierta manera los tres ejes centrales que diferencian, aunque en el fondo de forma convergente y/o complementaria, el desarrollo interno actual de la filosofía intercultural; como muestran, entre otras cosas, las actividades que coordinan y sus publicaciones ${ }^{17}$. A riesgo de resultar sumamente esquemático, pero la

\footnotetext{
${ }^{16}$ A título de ejemplo se pueden citar entre muchos otros: PANIKKAR Raimon, «Aporias in the Comparative Philosophy of Religión», en Man and World 3-4 (1980) 357-383; y Sobre el diálogo intercultural, Salamanca, 1990; FORNET-BETANCOURT Raúl, "Las relaciones raciales como problema de comprensión y comunicación intercultural. Hipótesis provisionales para una interpretación filosófica», en Cuadernos Americanos 18 (1989), 108-119 y Filosofía intercultural, México, 1994; HOLENSTEIN Elmar, Menschliches Selbstverständnis, Frankfurt, 1985; KIMMERLE Heinz, Philosophie in Afrika. Afrikanische Philosophie. Annäherung an einen interkulturellen Philosophiebegriff, Frankfurt, 1991; MALL Ram A/HÜLSMANN Heinz Hülsmann, Die drei Geburtsorte der Philosophie: China, Indien, Europa, Bonn, 1989; MORITZ Ralf Moritz/RÜSTAU Hiltrud/HOFFMANN Gerd R.(Hrsg.), Wie und warum entstand Philosophie in verschiedenen Regionen der Erde?, Berlin, 1988; ORUKA Henry O., Sage Philosophy. Indigenous Thinkers and Modern debate on African Philosophy, Leiden, 1990; WALDENFELS Bernhard, Der Stachel des Fremden, Frankfurt, 1990; y WIMMER Franz, Interkulturelle Philosophie. Geschichte und Theorie, Wien, 1990. Es evidente que me refiero aquí a autores vinculados a la «filosofía intercultural» en sentido explícito. Y lo aclaro porque el diálogo intercultural está más presente en la historia de la filosofía de lo que a primera vista aparece. De modo que, usando un criterio más amplio, se podría hacer referencia también a los «precursores» lejanos de un filosofar intercultural: Llull, Vico, Herder, etc.

17 También a título de ejemplo cabe recordar que la «Gesellschaft für Interkulturelle Philosophie» organiza desde 1992 Simposios internacionales y que desde 1993 edita la importante serie «Studien zur Interkulturellen Philosophie» en la que ya han aparecido 10 volúmenes. Por su parte la «Wiener Gesellschaft für Interkulturelle Philosophie» anima un intenso programa de conferencias y cursos en la universalidad de Viena y edita la revista Polylog. Zeitschrift für Interkulturelle Philosophien que es un verdadero foro internacional de discusión, documentación e información para todo el movimiento. Y el Departamento de América Latina del Missionswissenschaftliches Institut de Aachen, además de ser el centro coordinador de los Congresos Internacionales de Filosofía Intercultural, edita desde 1997 la serie «Denktraditionen im Dialog: Studien zur Befreiung und Interkulturalität» en la que ya se han publicado 26 tomos, entre ellos las actas de los seis congresos internacionales de filosofía intercultural celebrados hasta la fecha. Entre sus actividades hay que mencionar también la coordinación desde 1989 del diálogo intercultural norte-sur con la organización de once seminarios internacionales y la publicación de las actas correspondientes.
} 
limitación del espacio disponible hace inevitable correr este riesgo, las perspectivas del trabajo filosófico intercultural que se desarrollan desde dichos ejes centrales, pueden resumirse en los programas siguientes:

- El programa de una filosofía intercultural fundada sobre todo en la labor de interpretación y comprensión de las «superposiciones» culturales y que debería, por ello, cultivarse fundamentalmente como una actitud hermenéutica intercultural ${ }^{18}$.

- El programa de una filosofía intercultural entendida como reconstrucción de la historia de la filosofía desde la consulta de las distintas tradiciones de pensamiento de la humanidad y como desarrollo de un «polílogo» entre las muchas lenguas que habla la filosofía ${ }^{19}$.

- El programa de una filosofía intercultural como propuesta de una radical transformación de la filosofía desde el reconocimiento de sus fronteras actuales con otras formas del pensar, como por ejemplo la teología; y con la finalidad expresa de reconfigurar el quehacer filosófico como una actividad liberadora en el mundo de hoy ${ }^{20}$.

Estos tres programas, como decía, marcan hoy el ritmo del desarrollo de la filosofía intercultural. De manera que tomados en su conjunto, conforman el horizonte a cuya luz se pueden señalar (al menos provisionalmente, insisto) algunos alcances y límites de la filosofía intercultural. Sin poder entrar en más detalles ${ }^{21}$, acentuaré ahora el carácter complementario de sus perspectivas de trabajo para tomarlos como indicadores de un desarrollo complejo que permite resaltar ya ciertos alcances o logros. Serían, en lo esencial, éstos:

- Aportación a la corrección de la fragmentación posmoderna de la razón (filosófica) al fundar un nuevo modo de ver la pluralidad de las prácticas de la razón, a saber, comprendiéndolas como prácticas cuya pluralidad no se puede afirmar si no se reconoce al mismo tiempo la posibilidad de la relación entre las mismas. La contextualidad de la razón no produce «islas de razón» sino

\footnotetext{
${ }^{18}$ Cf. MALL Ram A., Philosophie im Vergleich der Kulturen, Darmstadt, 1995; y «Das Konzept einer interkulturellen Philosophie», en Polylog 1 (1998) 54-69.

${ }^{19}$ Cf. WIMMER Franz, Interkulturelle Philosophie, Wien, 990; y «Thesen, Bedingungen und Aufgaben interkulturell orientierter Philosophie», en Polylog 1 (1998) 5-12.

${ }^{20}$ Cf. PANIKKAR Raimon, Sobre el diálogo intercultural, Salamanca, 1990; "Filosofía y cultura: una relación problemática», en FORNET-BETANCOURT R. (Ed.), Kulturen der Philosophie. Dokumentation des I. Internationalen Kongresses für Internationale Philosophie, Aachen 1996, pp. 15-42; y «El imperativo cultural», en FORNET-BETANCOURT R. (Ed.), Unterwegs zur interkultureIlen Philosophie. Dokumentation des II. Internationalen Kongresses für Interkulturelle Philosophie, Frankfurt, 1998; pp. 20-42. FORNET-BETANCOURT Raúl, Transformación intercultural de la filosofía, Bilbao, 2001; y, como editor, Kulturen zwischen Tradition und Innovation. Stehen wir am Ende der traditionellen Kulturen? Dokumentation des III. Internationalen Kongresses für Interkulturelle Philosophie, Frankfurt, 2001.

${ }^{21}$ Para el análisis crítico de estas propuestas de filosofía intercultural me permito remitir a la obra citada de Diana de Vallescar.
} 
«zonas de traducción» donde el horizonte «racional» contextual de todos va creciendo precisamente por esa forma singular de relacionarse que es la traducción. De aquí:

- Contribución a la superación del relativismo cultural; ya que, al leer la pluralidad desde la clave de la relación, no deduce de la afirmación de la relatividad de las culturas ningún tipo de relativismo cultural sino más bien la necesidad de un proceso de relaciones que descentra toda cultura de sus fijaciones etnocéntricas y convierte de esta forma las diferencias culturales en diferencias históricas relativas ${ }^{22}$. En la relación de la traducción de su diferencia toda cultura descubre que no es «la medida» ni de sí misma ni de las otras.

- Promoción de un diálogo de culturas filosóficas que ha hecho posible entre otros aspectos- ver que el diagnóstico de Max Weber sobre el mundo moderno como un mundo regido por la racionalización y el «desencanto» (Entzauberung) es un diagnóstico de validez regional que no puede ser extrapolado sin más para explicar la dialéctica de tradición e innovación a escala universal.

- Fomento del desarrollo de filosofías contextuales conscientes de su diferencia cultural, pero que buscan la interacción entre sí y que interpelan a la tradición occidental dominante con el reclamo de entablar un diálogo en condiciones de igualdad ${ }^{23}$.

- Desarrollo de una hermenéutica de la alteridad que parte del reconocimiento del "extraño» como intérprete y traductor de su propia identidad, que supera el horizonte de la división «sujeto-objeto» y hace del trabajo hermenéutico un proceso de intercambio de interpretaciones, esto es, una tarea de comprensión participada y compartida entre intérpretes que, mediante ese proceso, van tomando cada vez más conciencia de lo que pierden cuando cierran su interpretación y se encierran en ella buscando una garantía estable para su identidad o tradiciones. Pues una de las enseñanzas fundamentales de la hermenéutica intercultural es la idea de que la comprensión profunda de lo que

\footnotetext{
${ }^{22}$ Cf. KETTNER Matthias, "Kulturrelativismus oder Kulturrelativität», en Dialektik 2 (2000) 17-38. Ver además: BARUZZI Arno/TAKEICHI Akihiro (Ed.), Ethos des Interkulturellen, Würzburg, 1998; BROCKER Manfred/NAU Heino (Ed.), Ethnozentrismus. Möglichkeiten und Grenzen des interkulturellen Dialogs, Darmstadt, 1997; y LÜTTERFELDS Wilhelm/MOHRS Thomas (Ed.), Eine Welt-eine Moral?, Darmstadt, 1997.

${ }^{23}$ Entre otros trabajos representativos cabe citar: ESTERMANN Josef, Filosofía andina. Estudio intercultural de la sabiduría autóctona andina, Quito, 1998; HOUNTONDJI Paulin (Ed.), Les savoirs endogènes, Dakar, 1994; LÖLKE Ulrich, Kritische Traditionen. Afrika als Ort der Dekolonisation, Frankfurt, 2001; NAGL-DOCEKAL Herta/WIMMER Franz M. (Ed.), Postkoloniales Philosophieren: Afrika, Wien/München, 1992; MADU Raphael O., African Symbols, Proverbs and Mythos, New York/Frankfurt, 1996; PAGANO Carlos M., Un modelo de filosofía intercultural: Rodolfo Kusch, Aachen, 1999; PANIKKAR Raimon, La experiencia filosófica de la India, Madrid, 1997; y PICOTTI Dina, El descubrimiento de América y la otredad de las culturas, Buenos Aires, 1990; y La presencia africana en nuestra identidad, Buenos Aires, 1998; y WAJOKI Gerald J., The Wisdom and Philosophy of the Gikuyu Proverbs, Nairobi, 1997.
} 
llamamos «propio» o «nuestro», es un proceso que requiere la participación interpretativa del otro $^{24}$.

- Contribución al desarrollo de una teoría política del «extranjero» que, inspirada en la hermenéutica intercultural, supera el horizonte de las prácticas políticas de muchos estados actuales que orientan sus políticas de extranjería en los principios de la asimilación y/o integración de los extranjeros en los correspondientes órdenes culturales, jurídicos, políticos, etc. vigentes para reclamar una política de autorización de los extranjeros que los reconoce como «iguales pero diferentes» y que por ello redefine interculturalmente las fronteras del orden jurídico-político establecido ${ }^{25}$.

- Aportación al análisis filosófico-crítico del fenómeno de la globalización neoliberal como una «cultura global» que amenaza con reducir al estado de «reservas» las Ilamadas culturales tradicionales y ante la cual debe afirmarse la voluntad ético-política de construir un mundo no globalizado, pero sí universalizado desde abajo con la participación solidaria de todos ${ }^{26}$.

Y en un nivel más concreto cabría mencionar todavía:

- La aportación a la superación en concreto del eurocentrismo fáctico que reina todavía en los planes de estudio de muchas facultades de filosofía al promover la creación de cátedras o cursos de filosofía intercultural en muchas universidades, como por ejemplo en Amsterdam, Bremen, México, Nairobi, Viena ${ }^{27}$.

\footnotetext{
${ }^{24}$ Ver las obras filosóficas citadas en este artículo, sobre todo en las notas 16, 18, 19 y 20.

${ }^{25}$ Además de las obras citadas en la nota 4 ver: KAUFFMANN Matthias (Ed.), Integration oder Toleranz, Freiburg, 2000; LEGGEWIE Claus, Multi-Kult. Spielregeln für die Vielvölkerrepublik, Berlin, 1993; el número monográfico «Ciudadanía e interculturalidad» de Revista Anthropos 191, (2001); OLIVÉ León (Coord.), Etica y diversidad cultural, México, 1993; y VILLORO Luis, Estado plural. Diversidad de culturas, México, 1998.

${ }^{26}$ Ver por ejemplo: BARTOLI Henri, «Mondialisation, hégémonie, et réactions identitaires», en Concordia 32 (1997) 41-54; BRIESKORN Norbert (Ed.), Globale Solidarität. Die verschiedenen Kulturen und die eine Welt, Stuttgart, 1997; COROMINAS Jordi, «Mundialización y acción liberadora», en Christus 703 (1997) 54-60; FORNET-BETANCOURT Raúl, «Tesis sobre la interculturalidad como alternativa a la globalización», en Asociación de Hispanismo Filosófico (Ed.), III. Jornadas de Hispanismo Filosófico, Santander, 1999, pp. 387-394; y, como editor, Kapitalistische Globalisierung und Befreiung, Frankfurt, 2000; SANTORI Anaya, "Un solo mundo. Responsabilidades y vínculos globales», en Estudios Centroamericanos 570 (1996) 307-326; y SCHWEPPENHÄUSER Gerhard/GLEITER Jörg H. (Ed.), Paradoxien der Globalisierung, Weimar 1999; pero también APEL Karl-Otto/HÖSLE Vittorio/SIMON-SCHÄFER Roland, Globalisierung. Herausforderung für die Philosophie, Bamberg, 1998; y TETZLAFF Rainer (Ed.), Weltkulturen unter Globaliserungsdruck, Bonn, 2000.

${ }^{27}$ Para la presentación de algunas de estas iniciativas ver: Polylog 1 (1998), 116-119. Ver también: BASU Sanchita/HÖHME-SERKE Evelyne/MACHER Maria (Ed.), Eurozentrismus: ¿Was gut ist, setzt sich durch? Beiträge zur Kritik einer die Welt beherrschenden Denk-und Handlungsweise, Frankfurt, 1999. Por otra parte debo indicar que el Missionswissenschaftliches Institut Missio en Aachen acaba de iniciar un proyecto internacional (en el que participan filósofos de Africa, Asia, Europa y América) que se propone precisamente elaborar una propuesta intercultural para la renovación de los planes de estudio en la enseñanza de la filosofía.
} 
Por lo que hace a los límites de la filosofía intercultural, me parece que hay que conceder que resulta muy difícil establecerlos hoy con claridad porque se trata de un modo de filosofar que está todavía en pleno desarrollo de sus potencialidades. No obstante, sobre el telón de fondo del desarrollo aquí resumido, intentaré enumerar brevemente algunos de los posibles límites o, mejor, «lagunas».

La más notoria es, sin duda, la ausencia de la perspectiva de género en el análisis filosófico intercultural que ha constatado con toda claridad Diana de Vallescar al señalar que "la racionalidad intercultural tiene un saldo pendiente respecto a la razón feminista ${ }^{28}$. Sin embargo, y esto muestra la provisionalidad de los límites hoy constatables, la misma autora de esta crítica constructiva está empeñada en el esfuerzo de amortizar ese «saldo pendiente» con la elaboración de un «proyecto genérico-intercultural-liberador» ${ }^{29}$.

Importante me parece igualmente la «laguna» en el análisis intercultural del concepto mismo de cultura, pues las ambivalencias en el manejo del término -que provienen, dicho sea de paso, sobre todo de que algunos representantes de la filosofía intercultural operan con una concepción un tanto esencialista de la cultura, pero quieren subrayar al mismo tiempo la interacción- han impedido elaborar una comprensión de las realidades culturales que, superando realmente las aproximaciones esencialistas, haga justicia a la historicidad de las mismas explicando las «leyendas» de sus supuestos mitos fundantes desde las prácticas históricas de hombres y mujeres concretos; y enseñando a distinguir entre el cultivo de la «imagen» de una cultura y su cultivo real en la vida de sus miembros.

Relacionado con el punto anterior está, para mí, lo que podría ser otro límite en los planteamientos actuales de la filosofía intercultural, a saber, la falta de un cuestionamiento explícito del tema del papel de la tradición o de las tradiciones culturales en la realidad de eso que llamamos, por ejemplo, "cultura alemana», "cultura española» o "cultura latinoamericana». Y no se trataría de explicar únicamente el proceso de constitución de tradición y/o tradiciones como eje generador de "cultura» sino también el uso, el modo de tratar y de relacionarse con ellas desde y en las prácticas culturales que podamos distinguir como tales, para aprender a distinguir entre «tradición» y realidad cultural sin fetichizar la «tradición», pero sin desactivarla tampoco. Pues entiendo que la elaboración de una explicación dialéctica de la diferencia entre ambas nos podría mostrar que ésta se produce en el marco de la relación histórica que genera de hecho toda práctica cultural.

\footnotetext{
${ }^{28}$ DE VALLESCAR Diana, op.cit.; p. 368 (cursiva en el original).

${ }^{29}$ Ibid., pp. 368-379. Este proyecto se desarrolla hoy en el marco de una investigación filosófica de largo aliento titulada «Interculturalidad y Mujer». También en el campo de la teología y de la pedagogía ya se trabaja por saldar esta hipoteca. Cf. AQUINO Pilar, "Theological Method in U.S. Latino/a Theology. Toward an Intercultural Theology for the Third Millennium», en ESPÍN Orlando O./DíAZ Miguel H. (Ed.), From the Heart of our People, New York, 1999, pp. 6-48; y KLESINGREMPEL Ursula, Género e interculturalidad, México -en prensa-.
} 
En este contexto sitúo también otra «laguna» en el discurso filosófico intercultural actual. Me refiero al tratamiento de la cuestión del reconocimiento de un «canon normativo» en las «culturas» y de sus consecuencias para el diálogo ente ellas. Pues, a pesar de las aportaciones señaladas a la superación del relativismo cultural, todavía está pendiente la reconstrucción intercultural de lo normativo y con ello también la fundamentación de los parámetros de una normatividad intercultural.

\section{Observación final}

Me permito terminar insistiendo en la provisionalidad de mi presentación de la filosofía intercultural porque, a la razón dada para justificar el carácter provisional de mi exposición -la «juventud» del movimiento- debo añadir que ello se explica, evidentemente, también por las limitaciones de la propia información $^{30}$. Aclarando esto, creo que, como resumen, se puede arriesgar la afirmación de que el futuro de la filosofía intercultural depende de su propia capacidad para activar todas sus dimensiones y articularse como paradigma que permita una nueva constelación del saber de la humanidad así como del ideal de lo que la humanidad realmente debe saber de cara a su justa realización como comunidad universal.

\footnotetext{
${ }^{30}$ Por eso remito de nuevo expresamente a la bibliografía citada. Ver además DEMENCHONOK Edward, «Intercultural Philosophy», en South Eastern Latin Americanist 4 (2001), 1-20.
} 